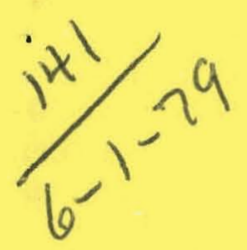

\title{
AVERAGE NEUTRALIZATION AND TRANSVERSE STABILITY IN ISABELLE
}

\author{
J. Herrera AND B. ZotTer
}

December 15, 1978

\section{ACCELERATOR DEPARTMENT}

BROOKHAVEN NATIONAL LABORATORY ASSOCIATED UNIVERSITIES, INC. UNDER CONTRACT NO, EY-76-C-02-0016 WITH THE UNITED STATES DEPARTMENT OF ENERGY 


\section{DISCLAIMER}

This report was prepared as an account of work sponsored by an agency of the United States Government. Neither the United States Government nor any agency Thereof, nor any of their employees, makes any warranty, express or implied, or assumes any legal liability or responsibility for the accuracy, completeness, or usefulness of any information, apparatus, product, or process disclosed, or represents that its use would not infringe privately owned rights. Reference herein to any specific commercial product, process, or service by trade name, trademark, manufacturer, or otherwise does not necessarily constitute or imply its endorsement, recommendation, or favoring by the United States Government or any agency thereof. The views and opinions of authors expressed herein do not necessarily state or reflect those of the United States Government or any agency thereof. 


\section{DISCLAIMER}

Portions of this document may be illegible in electronic image products. Images are produced from the best available original document. 


\title{
AVERAGE NEUTRALIZATION AND TRANSVERSE STABILITY IN ISABELLE
}

\author{
J. Herrera AND B. Zotter*
}

December 15, 1978

*Visiting Physicist. Permanent address CERN, Geneva, Switzerland.

\section{ACCELERATOR DEPARTMENT}

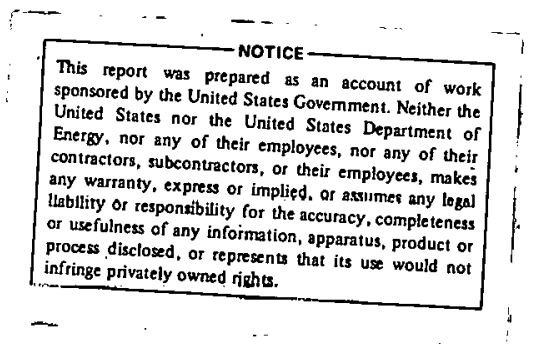




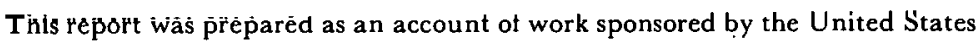
Government. Neither the United States nor the United States Department of Energy (DOE), nor any of their employees, nor any of their contractors, subcontractors, or their employees, makes any warranty, express or implied, or assumes any legal liability ar responsiluility fou lhe accursey, completenese or uefulneco of any information, apparatus, product or process disclosed, or represents that its use would not infringe privately owned rights.

Printed in the United States of America Available from

National Technical Information Service

U.S. Department of Commerce

5285 Port Royal Road

Springfield, VA 22161

Price: Printed Copy $\$ 4.00$; Microfiche $\$ 3.00$

March 1979

550 copies 


\begin{abstract}
clearing of electrons in the vicinity of the axis of a proton beam in the dipoles of a separated function AG-focusing structure is accomplished by transverse crossed-field drift due to the magnetic dipole field and a weak longitudinal electric field. This electric field is generated by potential differences caused by cross section variations of the beam due to variations of the $B$-functions and dispersion. The resulting radial drift brings the electrons into the off-axis region where they are driven out of the magnets by the usual longitudinal cross-field drift caused by the radial space charge field. The neutralization in ISABELLE is then a factor 20 below former estimates, and transverse stability can be obtained by chromaticity adjustments well within the design strengths of the sextupole correction windings.
\end{abstract}




\section{INTRODUCTION}

It has been pointed out recently that neutralization of the proton beam due to trapped electrons can lead to a coherent transverse instability ("brick wall-effect") in ISABELLE. 1 The planned strength of sextupole and/or octupole correction coils then would be insufficient to counteract the detuning. 2 These calculations are based on an estimate of neutralization 3 which establishes that a crossed-field drift, due to radial space charge fields, is the major contribution to clearing in the dipole magnets. Since the electric fields vanish at the beam axis, a small region around this axis remains uncleared and constitutes the main contribution to the expected average neutralization of slightly above $10^{-4}$ at high energy.

If the variations of the transverse beam dimensions due to changes of the $\beta$-functions combined with momentum dispersion are taken into account, we find that a longitudinal component of electric field of several $\mathrm{V} / \mathrm{m}$ also exists on the beam axis. This leads to a radial. drift of electrons across the otherwise uncleared region and the average neutralization is reduced to below $10^{-5}$. The remaining detuning of the working line is then sufficiently swall and can be corrected by sextupole/ octupole coils with less strength than foreseen and coherent transverse oscillation can be stabilized.

The longitudinal electric field on the beam axis will be modulated by the sagitta of the beam in the long dipoles. However, by properly centering the beams such that the excursions from the magnet-axis are minimized ( $5 \mathrm{~mm}$ for $5 \mathrm{~m}$ magnets) - which is also required for minimizing the available aperture - the effect becomes sufficiently small to be of no further consequence.

\section{BEAM POTENTIAL AND LONGITUDINAL ELECTRIC FIELD}

The potential of a uniform, cylindrical beam of charged particles (current $I_{0}$ ) of elliptic cross section (half width $w$, half height $h$ ) situated inside a circular cylindrical vacuum chamber (radius b) is given in Ref. 4. The rather complicated expression becomes quite simple for the center of a centrally located beam, where it can be written

$$
\Phi_{c}=\frac{Z_{o} I_{o}}{2 \pi}\left[1 \mathrm{u} \frac{2 b}{w+h}+\frac{1}{2}\right]
$$

$z_{0}=120 \pi(\Omega)$ is the free space impedance. If the beam is displaced from the chamber center (distance $d$ ), we find

$$
\Phi_{d}=\frac{z_{0} I_{0}}{2 \pi}\left[-\ln \frac{d(D+E)}{b(w+h)}+D \frac{D-E}{w^{2}-h^{2}}\right]
$$

1. G. Parzen, BNL Report, BNL 50842, ISA 78-5, (1978).

2. M. Month, G. Parzen, BNL Report, BNL 50843, ISA 78-6, (1978).

3. J.C. Herrera, BNL Report, BNL 50533, ISA 76-12, (1976).

4. E. Regenstreif, CERN Report, CERN/PS/DL-77-37, (1977). 
where

$$
\begin{aligned}
D & =\frac{b^{2}}{d}-d \\
E^{2} & =D^{2}-w^{2}+h^{2}
\end{aligned}
$$

and

Similar expressions can be found for the potentials at the beam edges, which are always less than the potential at the beam axis. To first order, the reduction of beam potential at the edge $x=w$ is given by

$$
\Phi_{c}-\Phi_{e}=\frac{Z_{o} I_{o}}{2 \pi} \frac{w}{w+h}
$$

If we neglect the fact that the beams in an AG-focusing machine are not quite cylindrical, we can evaluate the potentials at various positions along a Half-Cell of ISABELEE (see Table I)。

TABLE I. Potential in Beam Center (centered beam) For

$$
\begin{aligned}
& \epsilon_{\mathrm{H}}+\epsilon^{\mathrm{V}}=15 \pi 10^{-6}, \Delta \mathrm{p} / \mathrm{p}=2.6 \times 10^{-4}, \mathrm{Y}=426, \\
& \mathrm{~b}=44 \mathrm{~mm}, \mathrm{I}_{\mathrm{o}}=8 \mathrm{~A}
\end{aligned}
$$

\begin{tabular}{llllllll}
\hline Position & $\beta_{\mathrm{V}}(\mathrm{m})$ & $\beta_{\mathrm{H}}$ & $\mathrm{X}_{\mathrm{p}}(\mathrm{m})$ & $\mathrm{h}(\mathrm{mm})$ & $w(\mathrm{~mm})$ & $\Phi_{\mathrm{c}}(\mathrm{V})$ & $\Phi_{\text {edge }}(\mathrm{V})$ \\
\hline $\mathrm{QF}$ (center) & 11.5 & 66.7 & 2.73 & 0.318 & 1.476 & 2108.5 & 1713.7 \\
$\mathrm{BF}\left\{\begin{array}{l}\text { entrance } \\
\text { exit }\end{array}\right.$ & 13.2 & 59.6 & 2.58 & 0.341 & 1.395 & 2124.4 & 1738.7 \\
$\mathrm{~B} \quad$ entrance & 21.1 & 40.7 & 2.16 & 0.431 & 1.160 & 2166.2 & 1816.2 \\
lexit & 36.9 & 37.0 & 2.07 & 0.456 & 1.109 & 2174.2 & 1834.3 \\
$\mathrm{BD}\left\{\begin{array}{l}\text { entrance } \\
\text { exit }\end{array}\right.$ & 40.6 & 23.6 & 1.73 & 0.570 & 0.905 & 2202.4 & 1909.2 \\
$\mathrm{QD}$ (center) & 59.4 & 13.2 & 1.66 & 0.598 & 0.863 & 2207.0 & 1922.1 \\
\end{tabular}

Dividing the potential differences by the distances, we obtain an average longitudinal electric field on axis of about $6 \mathrm{~V} / \mathrm{m}(2.6,6.1$ and $9.1 \mathrm{~V} / \mathrm{m}$ in the three dipoles). The longitudinal electric fields are somewhat larger at the edges of the beam, but we will neglect this effect for simplicity.

Due to the sagitta of the beam in the long, straight dipoles, the center of the beam will be displaced less than the maximum value of

$$
d=\frac{L}{\varphi}\left(1-\cos \frac{\varphi}{2}\right) \approx \frac{\varphi L}{8}
$$

where $L$ is the length and $\varphi$ the bending angle, Reduction of the potential for a small displacement (and $\mathrm{w}^{2}-\mathrm{h}^{2}<<b^{2}$ ) can be found from Eqs. (1) and (2) and is approximated by

$$
\Delta \Phi=\Phi_{c}-\Phi_{d} \approx-\frac{z_{o} I_{o}}{2 \pi} \frac{d^{2}}{b^{2}}
$$

For ISABELLE dipoles with a length of $4.6 \mathrm{~m}$ and a bending angle of $17.2 \mathrm{mrad}$, the sagitta is almost $10 \mathrm{~mm}$, corresponding to a potential reduction of about $25 \mathrm{~V}$. Such a large reduction would cause a reversal of the 
longitudinal electric field. By centering the beam such that its maximum displacement is $\pm 5 \mathrm{~mm}$, the potential reduction is reduced to about $6 \mathrm{~V}$ and can be neglected (see Figure. 1).

\section{CROSSED FIELD DRIFT}

Due to the combined action of the longitudinal electric and the vertical magnetic field, the electrons will drift radially with a velocity given by

where

$$
\begin{gathered}
v_{x}=\frac{E_{v}}{B}=\frac{Z_{0} I_{0}}{2 \pi B L} S \\
S=\ln \frac{w_{1}+h_{1}}{w_{2}+h_{2}}
\end{gathered}
$$

and subscript 1 refers to the entrance, subscript 2 to the exit of a magnet of length $L$ and strength $B$. We neglect the initial velocity of electrons and the increase of longitudinal electric field from the axis, and integrate Eq. (7)。 We obtain

$$
\begin{aligned}
& X=X_{0}+s \frac{F}{L} t \\
& F=\frac{Z_{0} I_{0}}{2 \pi B}
\end{aligned}
$$

is written for short, and $x_{0}$ is the initial radial position of an electron. Once the electrons have drifted away from the axis they see the much stronger radial electric field due to space charge given by

$$
E_{X}=\frac{Z_{0} I_{0}}{2 \pi w^{2}} x
$$

where $w$ is the average half width of the beam. The longitudinal drift is then

$$
v_{Z}=-\frac{E x}{B}=-\frac{F}{w^{2}}\left(x_{0}+\frac{S F}{L} t\right),
$$

and a further integration with respect to time yields

$$
z=z_{0}-\frac{F}{w^{2}}\left(x_{0} t+\frac{S F}{2 L} \cdot t^{2}\right)
$$

For $S>0$ (positive longitudimal electric field), electrons with $\mathrm{X}_{\mathrm{o}}>0$ will travel to the left (decreasing $\mathrm{Z}$ ), while electrons with $x_{0}<0$ will at least initially travel to the right (see Figure 2). If $X_{0}$ is large enough in magnitude and negative $\left(X_{0}<-w \sqrt{25}\right)$ they will reach the magnet edge $Z=L$ and go to the nearest clearing 


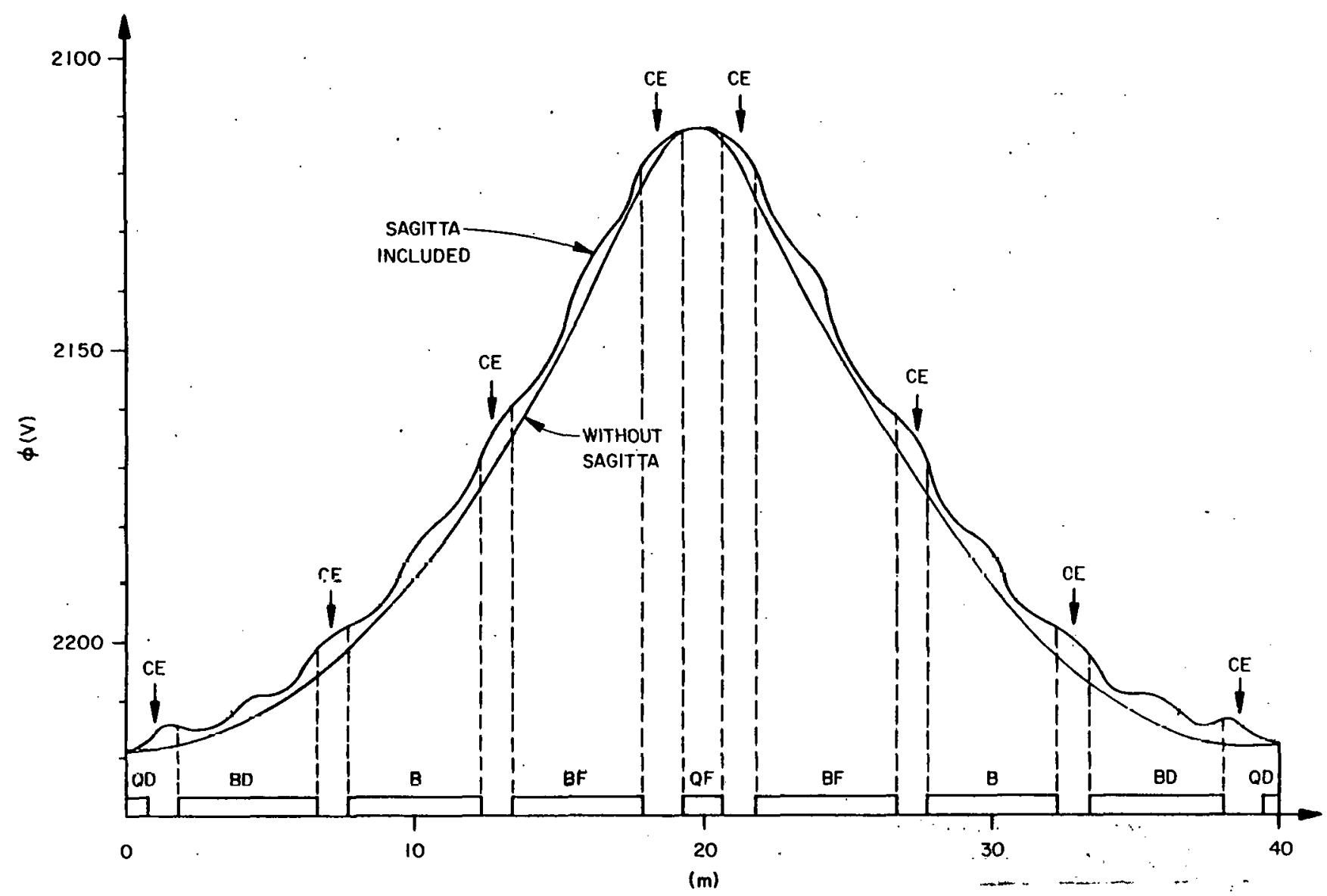

Figure 1. Potential at center of beam in Isabelle lattice cell.

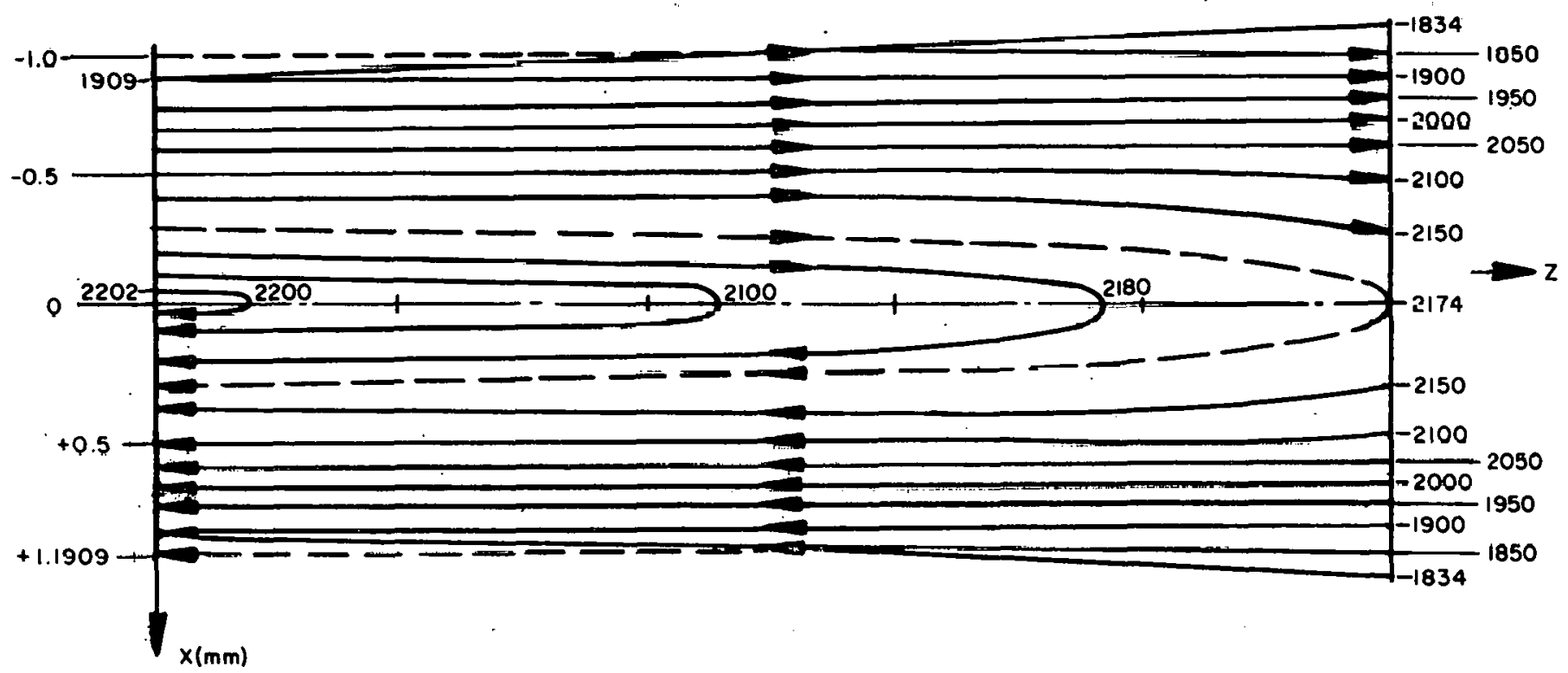

Figure 2. Beam cross-section, equi potentials and electron trajectories in dipole B (sagitta neglected). 
electrode. If $\mathrm{x}_{0}$ is less negative, they will get to the upper half of the magnet, reverse their direction and leave at $\mathrm{Z}=0$ 。 For $\mathrm{S}<0$, the whole picture is simply inverted.

We can consider Eq. (13) a quadratic for the traversal time $T$

$$
\mathrm{T}^{2}+\frac{2 \mathrm{LX}}{\mathrm{SF}} \mathrm{T}+\frac{2 \mathrm{LW}}{\mathrm{SF}^{2}} \quad\left(\mathrm{Z}-\mathrm{Z}_{\mathrm{o}}\right)=0
$$

with the solutions

$$
T=-\frac{L X_{o}}{S F}+\sqrt{\left(\frac{L X_{o}}{S F}\right)^{2}-\frac{2 L W}{S F^{2}}\left(Z-Z_{o}\right)}
$$

Assuming $S>0$, we have to take $\mathrm{Z}=0$ for $\mathrm{X}_{0}>0$. The longest traversal time is for particles with $Z_{0}=L$ and $X_{0}=0$ and becomes

$$
\mathrm{T}_{1}=\frac{\mathrm{WL}}{\mathrm{F}} \sqrt{\frac{2}{\mathrm{~S}}}
$$

For $\mathrm{x}_{0}<-\mathrm{w} \sqrt{2 \mathrm{~S}}$, we have to use $\mathrm{z}=\mathrm{L}$. The longest traversal time now is for particles with $\mathrm{Z}_{0}=0, \mathrm{X}_{0}=-\mathrm{w} \sqrt{25}$ and is again $\mathrm{T}_{1}$. For particles with $0<x_{0}<-w \sqrt{2 s}$, we have to use $z=0$, and the maximum traversal time is found for $z_{0}=0, x_{0}=-w \sqrt{2 \xi}$ and is

$$
\mathrm{T}_{\max }=2 \mathrm{~T}_{1}=\frac{2 \mathrm{WL}}{\mathrm{F}} \sqrt{\frac{2}{\mathrm{~S}}}
$$

For $\mathrm{S}<0$, we can use the same expression with $\mathrm{S}$ replaced by $|\mathrm{s}|$ in the squa re root.

\section{AVERAGE NEUTRALIZATION}

For equilibrium conditions, the number of electrons produced equals the number of electrons cleared

$$
R_{p} N_{p}=R_{c} N_{e}
$$

where $R_{p}$ is the clcctron production rate

$R_{C}^{p}$ is the electron clearing rate

$N_{p}$ the total number of protons

$\mathrm{N}_{\mathrm{e}}$ the total number of electrons.

The average neutralization is

$$
\eta=\frac{\mathrm{N}_{\mathrm{e}}}{\mathrm{N}_{\mathrm{p}}}=\frac{\mathrm{R}_{\mathrm{p}}}{\mathrm{R}_{\mathrm{c}}}
$$

The production rate has been calculated in Ref, 3 and is approximately $1.2 \times 10^{-2}$ for typical ISABELLE parameters. The clearing rate is the inverse of the traversal time, for which we take (on the conservative side) the maximum value given by $\mathrm{Eq}$. (17). Substituting for all abbreviations, 


$$
\eta=\left(\frac{4 \pi B L}{z_{o} I_{o}} R_{p}\right) w \sqrt{\frac{2}{\left|1 n \frac{w_{1}+\hbar_{1}}{w_{2}+h_{2}}\right|}}
$$

For $B=5 \mathrm{~T}, L=5 \mathrm{~m}, I_{0}=8 \mathrm{~A}$ the factor in parenthesis is about $1.25 \times 10^{-3}$. The actual values of the neutralization in the three lattice magnets of ISABELLE can be found in Table II,

TABLE II. Average Neutralization In Dipoles

$$
\mathrm{B}=5 \mathrm{~T}, \mathrm{~L}=5 \mathrm{~m}, \mathrm{I}_{\mathrm{o}}=8 \mathrm{~A}, \mathrm{R}_{\mathrm{p}}=1.2 \times 10^{-2}
$$

\begin{tabular}{llll}
\hline Magnet & $S=1 \mathrm{n} \frac{\mathrm{w}_{1}{ }^{+h} 1}{\mathrm{w}_{2}{ }^{+h} \mathrm{~h}_{2}}$ & $<w>(\mathrm{mm})$ & $\eta$ \\
\hline $\mathrm{BF}$ & 0.087 & 1.28 & $7.6 \times 10^{-6}$ \\
$\mathrm{~B}$ & 0.059 & 1.01 & $7.6 \times 10^{-6}$ \\
$\mathrm{BD}$ & 0.025 & 0.78 & $8.6 \times 10^{-6}$ \\
\hline
\end{tabular}

and are $8 \times 10^{-6}$ on average. Because we have neglected the reduction. of fields due to the sagitta of the beam, we shall use $\eta=10^{-5}$ as our estimate of the neutralization in the ISABELLE dipole magnet of high field. This value is 20 times smaller than the one previously used for estimating the sextupole and/or octupole fields required to correct the detuning of the working line. While the octupoles remain still marginal, the sextupoles should be more than sufficient for this purpose, provided the other regions in the machine can be cleared equally well. The neutralization in the long straight section in the insertions has been estimated to be about $3 \times 10^{-6}$, but this puts more stringent limitations on the cross section variations of the vacuum chamber wa11,5 and may necessitate an increase in the number of clearing electrodes in the field frec regione of IS $A$ BELLLE'。

\section{TRANSVERSE IMPEDANCE}

The transverse dispersion relation coefficients $U$ and $V$ are simply related to the tune shifts

$$
\mathrm{U}+(1-j) \mathrm{V}=\Omega_{0}\left(\Delta \nu_{\text {inc }}-\Delta \nu_{\text {coh }}\right)
$$

The tune shifts have been calculated for a wide variety of geometries, but generally under the assumption of perfectly conducting walls. They are usually expressed by the image coefficients $\xi$ and $\epsilon$ in the form

$$
\begin{aligned}
& \Delta \nu_{c o h}=-\frac{N \nu_{o} R}{\pi \beta^{2} \gamma \nu}\left[\frac{\varepsilon_{1}}{b^{2}}+\frac{\varepsilon_{1}}{\nu^{2} b^{2}}+\beta^{2} \frac{\epsilon_{2}}{g^{2}}\right] \\
& \left.\Delta \nu_{i n c}=-\frac{N \nu_{o} R}{\pi \beta^{2} \gamma \nu}-\frac{\epsilon_{1}}{b^{2}}+\frac{\epsilon^{2} 2}{g^{2}}+\frac{\epsilon_{s c}}{\gamma^{2} h^{2}}\right]
\end{aligned}
$$

5. J.C. Herrera, Proceedings 1976 ISABELLE Summer Workshops, Brookhaven, BNL 50611, 236 . 
where $\epsilon_{\mathrm{Sc}}=\mathrm{h} / \mathrm{h}+\mathrm{w}$ for elliptic beams,

$\varepsilon_{1}$ is the electrostatic,

$6_{2}$ is the magnetic,

$\xi_{1}$ is the coherent image coefficient,

$\mathrm{b}$ is the chamber radius,.

$\mathrm{g}$ is the pole-piece distance,

$R$ is the machine radius,

$r_{0}$ is the classical proton radius, and

$\mathrm{N}$ is the total number of protons.

In analogy to the longitudinal coupling impedance, it has become customary to define a transverse coupling impedance (per unit displacement, hence $\Omega / \mathrm{m})$, related to: $U$ : and $\mathrm{V}$ by

$$
z_{\perp}=j z_{0} \frac{2 \pi R \gamma \nu}{N_{\nu_{0}} \beta c}[U+(1-j) v]
$$

or, using Eqs. (20) - (22); we get

$$
z_{\perp}=j z \frac{2 R}{\beta^{2} \gamma^{2}}\left[\frac{\xi_{1}-\epsilon_{1}}{b^{2}}-\frac{\epsilon_{s c}}{h^{2}}\right]
$$

If we include the effect of neutralization, the coherent tune shift remains unchanged, while the electrostatic terms in the incoherent are reduced by $\eta$

$$
\Delta \nu_{\text {inc }}=-\frac{N_{\nu} R}{\pi \beta^{2} \gamma \nu}\left[(1-\eta) \frac{\varepsilon_{1}}{b^{2}}+\beta^{2} \frac{\varepsilon_{2}}{g^{2}}+\left(\frac{1}{v^{2}}-\eta\right) \frac{\epsilon_{s c}}{h^{2}}\right]
$$

and

$$
z_{\perp}=z_{\perp}^{\eta=0}+j z_{o} \frac{2 R \eta}{\beta^{2}}\left(\frac{\varepsilon_{1}}{b^{2}}+\frac{\varepsilon_{s c}}{h^{2}}\right)
$$

For $\eta \gamma^{2} \gg 1$ and $b \gg h$, the last term in Eq. (25) dominates and we find with $\mathrm{Eq} \cdot(23)$ and $\beta \approx 1$

$$
\mathrm{z}_{\perp} \approx \mathrm{j} \mathrm{z}_{\mathrm{o}} \frac{2 \mathrm{R} \eta}{\mathrm{h}(\mathrm{w}+\mathrm{h})}
$$

This value of $z_{\perp}$ increases with energy because $h$. and $w$. become smaller, and is directly proportional to the neutralization. For ISABELLE parameters $(\mathrm{R}=612 \mathrm{~m},\langle\mathrm{~h}\rangle=0.54 \mathrm{~mm},\langle\mathrm{w}\rangle=1.1 \mathrm{~mm})$ we find

$$
\mathrm{z}_{\perp}=5.3 \mathrm{j} \mathrm{M} \Omega / \mathrm{m} \text { (for } \eta=10^{-5} \text { ) }
$$

while it was over $100 \mathrm{j} M \Omega / m$ for $\eta=2 \times 10^{-4}$ used in Ref. 2 .

\section{STABILITY CRITÉRION}

Rather than using the exact criterion which requires $\left|z_{\perp}\right|<\overline{z_{\perp}} z_{\perp_{\text {max }}}$, where the form factor $\vec{F}$ depends on the distribution functions in momentum and/or amplitude as well as on the ratio of real to imaginary parts of $Z_{\perp}$, we use the simplified criterion that the frequency shift $\Delta w$ 
be less than the half-spread. $\delta$ w. The real frequency shift is equal to $(U+V)$, hence from Eq. (24) we find

$$
\Delta(\omega)=\frac{c^{I}}{4 \pi E_{0} / e} \cdot \frac{I m Z_{\perp}}{v \nu}
$$

while the frequency half-spread is

$$
\delta \omega=\left|x+(n-\nu) \eta_{p}\right| \Omega_{0}\left(\frac{\Delta p}{p}\right)_{\frac{1}{2}}
$$

where

$$
x=\frac{\mathrm{d} v}{\mathrm{dp} / \mathrm{p}} \text { is the chromaticity; }
$$

and

$$
\eta_{p}=\frac{d \Omega / \Omega}{d p / p} \text { the "frequency slip factor". }
$$

$\eta_{p}$ is positive above transition energy, hence, we want $x>0$ there in order to avoid cancellation of the two terms inside the absolute sign for the unstable modes $n>v$. We can then neglect the second term, which is usually quite small for the lowest mode numbers anyhow, and find the stability criterion

$$
\operatorname{ImZ_{\perp }}<4 \pi \frac{\mathrm{E}_{\mathrm{o}} \nu B \gamma}{\mathrm{eRI}}\left(\frac{\Delta \mathrm{p}}{\mathrm{p}}\right)_{\frac{1}{2}} x
$$

The RHS is essentia11y energy independent as $p / \beta Y=m_{0} c$ is an invariant. For ISABELLE parameters $\left(\nu=22.6, B_{V}=426, R=612 \mathrm{~m}, I_{0}=8 \mathrm{~A}\right.$, $\Delta \mathrm{p} / \mathrm{p}=2.6 \times 10^{-4}$ ) we obtain

$$
x>\frac{\operatorname{ImZ}}{6.10^{6}}=0.9
$$

For the former value of $\eta=2 \times 10^{-4}$ which $1 \mathrm{ed}$ to $\mathrm{z}_{\perp}>100 \mathrm{M} \Omega / \mathrm{m}$, we needed $x>17$ which was beyond the capabilities of the correction sextupoles. The present value is comfortably sma11, however, and correction we11 within the design strength of the sextupoles.

\section{Distribution: External}

